\title{
Analysis of KRAS and NRAS mutations in Greek patients with metastatic Colorectal Cancer (mCRC) on the registry of the Gastro-intestinal Cancer Study Group (GIC-SG)
}

Research Article

Nikolaos Gouvas ${ }^{1}$, Telenia Kalambaliki², Alexandra Voutsina ${ }^{3}$, Zenia Saridaki ${ }^{3}$,

Maria Tzardi ${ }^{4}$, Aristea Kalykaki², Maria Sfakianaki², Athanasios Athanasiadis ${ }^{5}$, Evaghelos Xynos ${ }^{6}$, loannis Boukovinas ${ }^{7}$, John Souglakos²*

${ }^{1}$ Colorectal department, Worcester Acute Hospitals NHS Trust, Worcester, UK

2Laboratory of translational cancer research, University of Crete, Heraklion, Greece

3Medical Oncology Department, Asklepios Hospital, Heraklion, Greece

${ }^{4}$ Department of Pathology, University of Crete, Heraklion, Greece

${ }^{5}$ Department of Medical Oncology, General Hospital of Larissa, Larissa, Greece

${ }^{6}$ Colorectal department, InterClinic Hospital, Heraklion, Greece

'Department of Medical Oncology, Bioclinic Hospital, Thessaloniki, Greece

Received 22 March 2018; Accepted 17 March 2019

\begin{abstract}
Introduction: Several studies show that mutational profiles could influence treatment decisions in patients with metastatic CRC (mCRC). KRAS mutational status was the first step in biomarkers development in the era of molecular targeted therapies. Recently, NRAS mutational status was identified as an independent prognostic factor for the response to treatment with anti-EGFR moAbs. The aim of this observational study was to assess the feasibility of the KRAS/NRAS mutational analysis in patients with metastatic colorectal cancer in Greece and to identify any correlations with known clinical characteristics and histopathologic features. Methods: From January 2014 until September 2014 all patients registered to the GIC-SG database with newly diagnosed metastatic disease fromcolonorrectalcancerwereincludedandtumorsampleswereanalyzedforkras/nrasmutationsin 9 differentcertifiedlaboratoriesin Greece. Results: Samples from 510 patients were analyzed. Mutations' distribution was as follows: 173 (33,9\%) KRAS exon 2, 10 (2\%) KRAS exon 3, 25 (4,9\%) KRAS exon 4, 22 (4,3\%) NRAS exon 2, 11 (2,2\%) NRAS exon 3 and $3(0,6 \%)$ NRAS exon 4 . The only factor significantly associated with RAS mutational status was primary tumor location, with right sided tumors exhibiting higher rates of mutations. Discussion: The incidence and distribution of KRAS or NRAS exon 2-4 mutations are in accordance with those reported in the literature. The most significant clinical or pathological parameter revealed from the analysis is the location of the primary tumor.
\end{abstract}

Keywords: KRAS-NRAS mutation • colorectal cancer • metastatic disease

\section{Introduction}

Mounting knowledge of colorectal cancer (CRC) molecular genetics has substantially changed our approach towards the treatment of the disease, particularly in the metastatic setting. Up to date, several studies have shown that mutation profiles could influence treatment decisions in patients with metastatic CRC (mCRC). The determination of KRAS mutational status was the first step in the biomarkers development in the era of molecular targeted therapies. It has been proven that patients with mutations in exon 2, codons 12 and 13 of the KRAS oncogene gain no benefit from treatment with monoclonal antibodies (moAbs) against Epidermal Growth Factor Receptor (EGFR), cetuximab and panitumumab [1, 2]. But, beyond KRAS exon 2 mutations, the clinical relevance of other proposed mechanism of resistance to anti-EGFR moAbs, such as, other KRAS mutations outside codons 12 and 13 and mutations in other RAS gene family members, such as NRAS, have been also established later as biomarkers for selection of patients having the higher probability of response to this kind of treatment. Since most available data come from retrospective studies, validation in prospective trials 
is imperative. Although anti-EGFR moAbs cetuximab and panitumumab were initially registered for patients whose tumors were found to immunohistochemically express the EGFR protein, it soon became clear that this methodology was neither enough nor adequate to predict treatment efficacy. The activation of a growth factor receptor induces the recruitment of K-ras, which initiates the activation of a cascade of serine-threonine kinases leading to the signal transduction from the cell surface to the nucleus. KRAS mutations are present in more than one third of CRCs and in more than $90 \%$ of the cases they are located in exon 2, (codons 12 and 13) [1, 2]. KRAS mutations lead to the activation of one of the most important pathways for cell proliferation, the Ras/MAPK pathway, by inducing cyclin D1 synthesis. Consequently, in the presence of a KRAS mutation this activation pathway cannot be significantly inhibited by an anti-EGFR moAb (cetuximab or panitumumab) which acts upstream of the K-ras protein.

There is substantial evidence that specific KRAS mutations in hotspots located in codons 12 and 13 were predictors of resistance to anti-EGFR moAbs therapy and were associated with reduced efficacy and shorter progression free and overall survival [3, 4]. Apart from the fact that the majority of KRAS mutations which are located in exon 2 (codons 12 and 13), others exist, accounting approximately for $10 \%$ of the KRAS mutated cases and found mainly in exon 3 (codon 61) and 4 (codon 146). Preclinical data indicate that the above mentioned mutations result in phenotypes similar to the hotspot mutations leading to the RAS-RAF-MAPK kinase pathway activation. In contrast, other KRAS mutations, such as those found in codon 19 and 164 are phenotypically equivalent to wt KRAS [5, 6].

Similarly, NRAS encodes for a protein-member of the RAS superfamily of GTPases, and, like the rest of them plays a key role in the critical MAPK signaling pathway. Besides CRC, NRAS mutations are found in solid tumors like melanoma and thyroid cancer [7, 8]. Approximately $80 \%$ of the mutations reported in the NRAS gene are located in codon 61 [7, 9]. Again, in retrospective analysis, it was shown that patients with KRAS and BRAF wt but NRAS mutated tumors had significantly reduced response rates when treated with cetuximab in the salvage treatment setting [6]. Furthermore, in the retrospective analysis of panitumumab registration trial it was shown that panitumumab had no effect in KRAS wt but NRAS mutated patients [10]. The analysis of NRAS mutations is now mandatory for the administration of anti-EGFR moAbs for the treatment of patients with metastatic CRC.

The aim of this observational study was to assess the feasibility of the KRAS/NRAS exons 2 (codons 12,
13), 3(codon 61), 4 (codons 117 and 146) mutational analysis in patients with metastatic colorectal cancer in Greece as part of the daily clinical practice and to identify any correlations of RAS mutational status with known clinical characteristics and histopathologic features.

\section{Methods}

From January 2014 until September 2014 all patients registered to the GIC-SG database with newly diagnosed metastatic disease from colon or rectal cancer were included in the analysis. KRAS exon 2 (codons 12, 13) exon 3 (codon 61) and exon 4 (codons 117 and 146) and NRAS exon 2 (codons 12,13) exon 3 (codon 61) and exon 4 (codons 117 and 146) mutational analysis has been performed in 9 certified laboratories across Greece. Each laboratory was responsible for the mutational analysis of a specific geographical area, in order to reduce the time required for the analysis as well as the cost. The mutational analysis and report was systematically performed according to the consensus guidelines of the Hellenic Society of Medical Oncology (FCO 2014).

Samples' registration and all the logistics of the study were performed by the Hellenic Society of Medical Oncology (HeSMO). Data management and analysis were performed by the Gastrolntestinal Cancer Study Group (GIC-SG). Amgen Hellas supported the cost for the mutational analysis through a research grant to the GIC-SG.

Patients with histologically confirmed metastatic CRC aged above 18 years were enrolled in a web-based registry developed by the GIC-SG, which included all clinical parameters and relevant histopathological features of the tumors. All patients in order to be eligible for the registry had to sign an informed consent.

\section{Statistics}

Due to the descriptive nature of the study no formal sample size calculation was undertaken. Using the normal approximation method a sample size of 500 (95\% ci: 467-528) patients was considered to be sufficient for the analysis of the primary and secondary endpoints. The prevalence of mutations in the study population was anticipated to be the follow:

KRAS exon 2 (codon 12,13) 42\% (95\% Cl: 36.8\%47.6\%)

KRAS exon 3 (codon 61) 2.2\% (95\% Cl: 1.4\%-3.2\%)

KRAS exon 4 (codon 117 and 146) 4.8\% (95\% Cl: $3.5 \%-6.4 \%$ ) 
NRAS exon 2 (codon 12,13) 2.1\% (95\% Cl: 1.4\%$3.3 \%$ )

KRAS exon 3 (codon 61) 2.5\% (95\% Cl: 1.7\%-3.3\%)

KRAS exon 4 (codon 117 and 146) 0.8\% (95\% Cl: $0.0 \%-1.4 \%)$

Descriptive statistics are used for summaries of study variable (means, medians, percentages etc) and the results are presented in tables as appropriate. Associations between baseline characteristics and response were compared with two-sided Fisher's exact test or the Chi-square test for categorical variables and the Kruskal-Wallis test for continuous variables.

\section{Results}

Five hundred and fourteen patients were enrolled in the study. Appropriate tumor samples after pathology review were submitted for analysis of KRAS-NRAS mutations. Samples from four patients were not appropriate for analysis. At the end, samples from 510 patients were analyzed. The median time from the registration to analysis was 10 calendar days (range 5-13 days) or 7 working days (5-12 days), while the cost of the analysis for all 6 exons was $250 €$.

Patients and disease features are presented in Table 1. In brief 329 male patients (65\%) and 181 females $(35 \%)$ were enrolled in the study. The median age for the whole study population was 64 years, while a hundred and thirty patients were above 70 years old at the time of the analysis $(25,5 \%)$ whereas the other $380(74,5 \%)$ were below that age. The distribution of the primary tumors along the bowel was as follows: 169 (33\%) patients had their tumor at the right colon, 215 $(42 \%)$ at the left colon and $126(24 \%)$ at the rectum. As far as the localization of the metastatic disease is concerned, $379(74 \%)$ patients had metastases in the liver, lung metastases were found in 193 (37\%) patients and peritoneal disease was evident in 98 (19\%). Two hundred and eighty-eight patients (56\%) had synchronous metastatic disease and the rest 222 (43\%) developed metastases metachronously. The grading of the tumors analyzed was unknown for 75 (14\%) patients whereas $323(63 \%)$ patients had a well- or moderatedifferentiated tumor and the rest $112(22 \%)$ were poorly differentiated. Lastly, 85 (16\%) patients had mucinous tumors whereas 351 (68\%) were not mucinous. In 74 $(14 \%)$ patients it was not possible to identify correctly the mucinous part so their status was allocated as unknown.

Mutations to the RAS family genes were identified in tumors from 244 patients $(47.8 \%$ ), whereas the rest 266
Table 1: Patients and disease characteristics

\begin{tabular}{|c|c|c|c|}
\hline Feature & & $\begin{array}{c}\text { Patients } \\
\#\end{array}$ & $\%$ \\
\hline \multirow[t]{3}{*}{ Gender } & & 510 & 100 \\
\hline & Male & 329 & 65 \\
\hline & Female & 181 & 35 \\
\hline \multicolumn{4}{|c|}{ Tumor Location } \\
\hline & Right Colon & 169 & 33 \\
\hline & Left Colon & 215 & 42 \\
\hline & Rectum & 126 & 25 \\
\hline \multicolumn{4}{|c|}{$\begin{array}{l}\text { Tumor } \\
\text { Differentiation }\end{array}$} \\
\hline & Well/Moderate & 323 & 63 \\
\hline & Undifferentiated & 112 & 22 \\
\hline & Unknown & 74 & 14 \\
\hline \multirow[t]{3}{*}{ Mucinous } & Yes & 351 & 69 \\
\hline & No & 85 & 17 \\
\hline & Unknown & 74 & 14 \\
\hline \multicolumn{4}{|c|}{ Metastatic sites } \\
\hline & Liver & 379 & 74 \\
\hline & Lung & 193 & 38 \\
\hline & Peritoneum & 98 & 19 \\
\hline \multicolumn{4}{|l|}{ Mutation } \\
\hline & Present & 244 & 48 \\
\hline & Undetectable & 256 & 52 \\
\hline
\end{tabular}

(52.2\%) had tumors with undetectable RAS mutations (Figures 1 and 2). Mutations were distributed among the different exons of the KRAS-NRAS gene family as follows: 173 (33,9\%) KRAS exon 2, 10 (2\%) KRAS exon 3, 25 (4,9\%) KRAS exon 4, 22 (4,3\%) NRAS exon 2, 11 $(2,2 \%)$ NRAS exon 3 and $3(0,6 \%)$ NRAS exon 4 . There was no statistical difference for the detection rates among the 9 different laboratories where the analyses were performed.

There was no significant association between the RAS mutational status and the gender $(p=0.095)$ or the age of the patients $(p=0.360)$. The histopathological characteristics of the tumors (mucinous tumors, grade) did not exhibit any statistically significant association with the mutational status of the RAS gene family. On the other hand, there was a significant association between 
Table 2: Correlation of RAS mutation with clinical and pathological characteristics

\begin{tabular}{|c|c|c|c|c|c|}
\hline \multicolumn{3}{|l|}{ Feature No (\%) } & \multicolumn{2}{|c|}{ RAS mutation } & \multirow[t]{2}{*}{$p$ value } \\
\hline & & Total & Wild Type & Mutant & \\
\hline \multirow{2}{*}{ Age } & $\leq 70$ years & $380(75)$ & $203(53.4)$ & $177(46.6)$ & \multirow{2}{*}{0.360} \\
\hline & $>70$ years & $130(25)$ & $63(48.5)$ & $67(51.5)$ & \\
\hline \multirow{2}{*}{ Tumor Differentiation } & Low grade & $323(74)$ & $159(49.2)$ & $164(50.8)$ & \multirow{2}{*}{0.444} \\
\hline & High grade & $112(26)$ & $60(53.6)$ & $52(46.4)$ & \\
\hline \multirow{2}{*}{ Tumor Location } & Right & 169 (33) & 108 (63.9) & 61 (36.9) & \multirow{2}{*}{$<0.001$} \\
\hline & Left & $341(67)$ & 136 (39.9) & $205(77.1)$ & \\
\hline \multirow{2}{*}{ Mucinous } & Yes & $85(17)$ & $49(57.6)$ & $36(42.4)$ & \multirow{2}{*}{0.187} \\
\hline & No & $351(83)$ & $173(49.3)$ & $178(50.7)$ & \\
\hline \multirow{2}{*}{ Gender } & Male & $389(65)$ & $148(45)$ & $181(55)$ & \multirow{2}{*}{0.095} \\
\hline & Female & $181(35)$ & $96(53)$ & $85(45)$ & \\
\hline
\end{tabular}

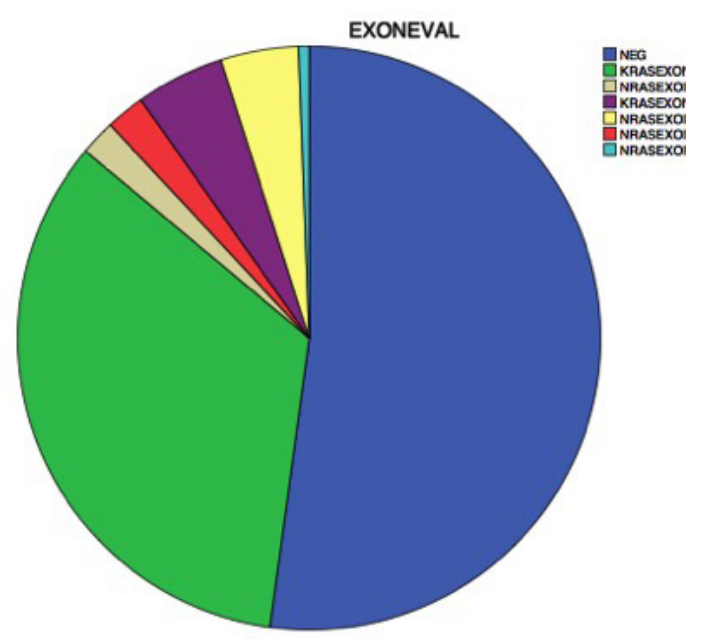

Figure 1: Distribution of KRAS and NRAS mutations by exon in all 510 included patients.

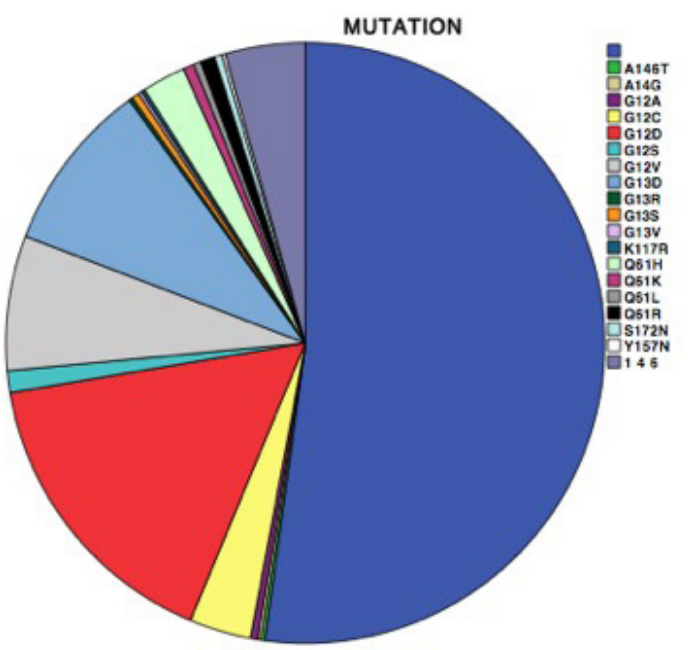

Figure 2. Distribution of KRAS and NRAS mutations by specific mutation in all 510 included patients.
RAS mutational status and the location of the primary tumor in the bowel $(p<0.0001)$ with right-sided tumors exhibiting significantly higher rates of mutations (108 out of $169-63,9 \%$ patients samples with detectable mutations), whereas left-sided tumors (left colon and rectum) showed significantly lower rates (136 out of 341 - 39,9\% patients samples with detectable mutations). Multifactorial analysis revealed also the location of the primary tumor as the only independent factor for the detection of a RAS mutation ( $p$ value $<0.0001$ ). No difference between KRAS or NRAS mutations detection was observed regarding tumor location (interactive $p$ value $=0.326$ )

\section{Discussion}

Firstly, the present study represents a successful example for the fruitful collaboration between a national research group, a national association of medical oncology and a pharmaceutical company. With this collaboration it became possible to tackle a significant problem in the daily clinical practice in the middle of severe economic crisis.

In details, the significant predictive role of beyond exon 2 KRAS mutations as well as those of the other member of the family NRAS, for the selection of patients with $\mathrm{mCRC}$ who will benefit the most from the anti-EGFR moAbs treatment, was first reported and published in June 2013 [10]. That led to the reconsideration of the reimbursement requirements from both cetuximab and panitumumab from EMEA and FDA, making the analysis of exon 2-4 KRAS and NRAS mutations mandatory for the prescription of these two drugs. Although the oncologic community had accept this step forward with great enthusiasm, the national authorities were 
unprepared to provide an effective and reliable network for the systemic analysis of exon 2-4 KRAS and NRAS mutations.

The GIC-SG took the initiative to conduct a prospective analysis of RAS mutations in patients with newly diagnosed metastatic colorectal cancer, registered in a database after the $1^{\text {st }}$ January 2014 . The GIC-SG was able to include 510 patients in a period of 8 months, reflecting the increase interest of the Greek oncologic community for the upfront analysis for RAS mutations at the time of presentation of metastatic disease in patients with colorectal cancer. The study along with a consensus meeting conducted by the HeSMO has led to the approval of the reimbursement of RAS testing for patients with metastatic colorectal cancer in Greece among other genetic tests required for the reimbursement of targeted therapies (i.e. EGFR mutations in Non-Small Cell Lung Cancer).

The incidence and distribution of KRAS or NRAS exon 2-4 mutations are in accordance with those reported in the literature [10]. The most significant clinical or pathological parameter revealed from the analysis is the location of the primary tumor[11]. Indeed other studies recently emphasize the importance of tumor location

\section{References}

[1] Amado RG, Wolf $M$, Peeters $M$, et al. Wild-type KRAS is required for panitumumab efficacy in patients with metastatic colorectal cancer. J Clin Oncol 2008;26:1626-1634.

[2] Karapetis CS, Khambata-Ford S, Jonker DJ, et al. K-ras mutations and benefit from cetuximab in advanced colorectal cancer. $N$ Engl $J$ Med 2008;359:1757-1765.

[3] Peeters M, Douillard JY, Van Cutsem E, et al. Mutant KRAS codon 12 and 13 alleles in patients with metastatic colorectal cancer: assessment as prognostic and predictive biomarkers of response to panitumumab. J Clin Oncol 2013;31:759-765.

[4] Van Cutsem E, Kohne CH, Hitre E, et al. Cetuximab and chemotherapy as initial treatment for metastatic colorectal cancer. N Engl J Med 2009;360:14081417.

[5] Loupakis F, Ruzzo A, Cremolini C, et al. KRAS codon 61, 146 and BRAF mutations predict resistance to cetuximab plus irinotecan in KRAS codon 12 and 13 wild-type metastatic colorectal cancer. Br J Cancer 2009;101:715-721.

[6] Lurkin I, Stoehr R, Hurst CD, et al. Two multiplex assays that simultaneously identify 22 possible mutation sites in the KRAS, BRAF, NRAS and PIK3CA genes. PLoS One 2010;5:e8802. for the selection of the most appropriate treatment [12]. According to these findings tumors located in the right colon (caecum, ascending colon and right flexure) bear more frequently KRAS-NRAS mutations, all well as BRAF mutations, in comparison with those located in the left colon (sigmoid colon, descending colon and left flexure) and this affects the response to anti-EFGR moAbs [12]. In contrast other clinical (such as gender, age, number of affected organs) and pathological parameters (such as histological grade and mutinous features) have no significant association with specific mutational detection rate.

The major weakness of the present study was the fact that mutational analysis was undertaken by different laboratories using different methods. On the other hand, all laboratories included in the study were certified for the KRAS and NRAS analysis and followed strict criteria for the analysis and report of predictive biomarkers as those were described in a consensus meeting of HeSMO. Despite that the findings of the study should be used as a hypothesis, the study generated results which may lead to further clinical and translational investigations and this is exactly the future plan of the GIC-SG.

[7] Hacker E, Nagore E, Cerroni L, et al. NRAS and BRAF mutations in cutaneous melanoma and the association with MC1R genotype: findings from Spanish and Austrian populations. J Invest Dermatol 2013;133:1027-1033.

[8] Park SJ, Sun JY, Hong K, et al. Application of BRAF, NRAS, KRAS mutations as markers for the detection of papillary thyroid cancer from FNAB specimens by pyrosequencing analysis. Clin Chem Lab Med 2013;51:1673-1680.

[9] Lee JH, Choi JW, Kim YS. Frequencies of BRAF and NRAS mutations are different in histological types and sites of origin of cutaneous melanoma: a meta-analysis. Br J Dermatol 2011;164:776-784.

[10] Oliner KSD, J.Y.; Siena,S.; Tabernero,J.; Burker,M. Analysis of KRAS/NRAS and BRAF Mutations in the Phase 3 Study of Panitumumab (pmab) + FOLFOX vs FOLFOX as 1st-Line Treatment (tx) for Metastatic Colorectal Cancer (mCRC). in J Clin Oncol. 2013.

[11] Missiaglia E, Jacobs B, D'Ario G, et al. Distal and proximal colon cancers differ in terms of molecular, pathological, and clinical features. Ann Oncol 2014;25:1995-2001.

[12] Heinemann VM, P.D.; von Weikersthal, L.F.; Decker, T.; Kiani, A.; Vehling-Kaiser, U.; Al-Batran, 
S.; Heintges, T.; Lerchenmuller, C.A.; Kahl, C.; Seipelt, G.; Kullmann, F.; Stauch, M.; Scheithauer, W.; Held, S.; Giessen, C.A.; Jung, A.; Kirchner, T.; Stintzing, S. Gender and tumor location as predictors for efficacy: Influence on endpoints in first-line treatment with FOLFIRI in combination with cetuximab or bevacizumab in the AIO KRK 0306 (FIRE3) trial. J Clin Oncol 2014;32. 